\title{
Adaptive Motion Control in Uncertain Environments using Tactile Feedback
}

\author{
Felix Sygulla, Christoph Schuetz and Daniel Rixen
}

\begin{abstract}
Next generation robot applications are expected to leave the field of complex tasks in simple environments and move on to simple and complex tasks in complex environments. In our opinion, tactile feedback is a key technology for motion planning in such unstructured environments as visual information may be insufficient or even unavailable. In this paper, we show the performance of a tactile feedback controller in jointspace, which is not bound to the null space of the manipulator. Additionally, we extend our tactile feedback control framework to hierarchical multi-space controllers with adaptive prioritization. This allows to dissolve the trade-off between low contact forces and good positional tracking and aims at applications, where desired trajectories must be held using manipulator redundancy and end-effector deviation is only admissible at high contact forces. The stability of this approach is discussed as well. Furthermore, we present an online stiffness estimation algorithm to increase the performance of our controllers in uncertain environments. Several real-world experiments with a 9-DOF multipurpose manipulator in collision with soft and hard objects show the capability of our work.
\end{abstract}

\section{INTRODUCTION}

Robots are expected to expand into plenty of new applications as well as our daily life. Their use is no longer restricted to segregated areas as known from traditional industrial manufacturing, but they will share their workspace with livings like humans, animals, plants and many other potential obstacles. They face surroundings, where the traditional assumption in robot motion planning of rigid and fixed obstacles no longer holds, where objects can be soft or hard, movable or fixed, deformable or rigid, elastic or stiff, while always being sensitive to force limits. Mechanical properties matter in these novel, unstructured, cluttered environments and equipping the robots with tactile sensing capabilities is obvious and inevitable.

The desired behavior of the robots can be pictured as follows: A task is given to the robot and is being started immediately. When a contact with the environment occurs, the robot pursues the task while exploiting its redundancy to reduce contact forces. In case a certain force limit is reached, the robot might abandon the task, but only as long and as far as needed in order to keep track of the force limit. We aim at teaching this behavior to a position-controlled robot with a tactile sensing module. It shall be enabled to interact with unmodeled environments in a deterministic manner while always respecting admissible contact force limits.

F. Sygulla, C. Schuetz and D. Rixen are with the Institute of Applied Mechanics, Technical University of Munich, Boltzmannstr. 15, 85748 Garching, Germany ffelix.sygulla, christoph.schuetz, rixen\}@tum.de
In our previous publication [1] we introduced a general tactile planning framework and conducted preliminary experiments using only a small subset of control options. Our new contribution extends this framework significantly and implements capabilities that only have been indicated before. The major topics and structure of this paper can be summarized as follows: In Sec. IV, we introduce a Joint Space Controller, which modifies the manipulator's motion directly in terms of joint-velocities. Furthermore, we present a hierarchical Multispace-Controller with adaptive prioritization in Sec. V, where contact forces are lowered exploiting the null space while the task is modified above some force threshold. Additionally, a high-performance controller design requires knowledge about the environment's stiffness: Thus, we present an online stiffness estimation algorithm in Sec. VI, which proves its capabilities in scenarios with obstacles of wide-range stiffnesses. A detailed presentation of our real-world experiments in Sec. VII as well as a critical discussion concludes our paper.

\section{RELATED WORK}

Within this section, we present several related approaches for motion planning, force control, and stiffness estimation and put them in the context of our contribution.

\section{A. Motion Planning in Uncertain Environments}

There are several commonly-known approaches for motion planning with obstacle avoidance in structured environments, [2]-[6]. However, these methods require prior knowledge about the position and geometry of objects and try to generate collision-free trajectories by all means. In unstructured and highly cluttered environments, a collision-free trajectory may simply not exist and contact with the environment may be inevitable for a given task. There have been investigations on motion-planning with rearrangeable [7], [8] and deformable [9], [10] objects, but these algorithms still require prior knowledge about location and mechanical properties. With tactile feedback, the properties of objects can be inferred simply by touch. Furthermore, motion planning in deeply cluttered environments, where the line of sight may be obscured and thus vision-based systems may fail, is enabled by this technique.

Some research has been made on instantaneous reactions to the detection of collisions with previously unknown objects, [11]-[13], but the contact force is not directly controlled or limited. Furthermore, only contacts between the end-effector and the environment are considered. 
In [14], a tactile skin on a manipulator arm is used to reach goal locations in deeply cluttered environments. The approach implements a simple impedance control and MPClike scheme with time-horizon of one time step. The search for a feasible goal location at low contact forces is described as a QP with resulting high computational effort. Compared to the locally optimal approach in our work, which runs in a $1 \mathrm{~ms}$ loop, the sample rates between $10-20 \mathrm{~ms}$ are significantly higher. Furthermore, no explicit utilization of the manipulator null space with hierarchical prioritization of end-effector tasks is performed and parameter selection may be difficult. The approach has been extended to a multi-step horizon with consideration of the robot dynamics as well as a global planning algorithm, [15], [16].

\section{B. Force Control}

Our tactile feedback approach is closely related to the force control domain, which has already been extensively explored. Indirect force control schemes, such as impedance and admittance control do not track a desired contact force, but aim to reach a certain mechanical contact behavior. Direct force control such as hybrid force/motion control is designed to track desired force and motion in separated spaces. For an overview of force control, refer to [17]. The classical force control approaches do only consider contact with the end-effector, yet have also been applied to general contact locations [18]. Still, these approaches do not separate between null space and task-space movements or use adaptive prioritization of the tasks.

In [19], a framework for multiple contact scenarios based on hybrid force/motion control is introduced. Motion and force control spaces are separated by the redundancy of the manipulator. In contrast to our work, the motion has lower priority and is projected in the null space of explicit force control. [20] proposes a control scheme based on hybrid impedance control, which allows to specify different dynamics for task-space and null space. Only the damping can be different though, and the parameter selection is difficult. The prioritized multi-task approach in [21] allows for hierarchical compliant control of torque-controlled redundant manipulators. Different impedance parameters can be specified for task- and null space. There also exists work on multiple-priority impedance control schemes with utilization of different control spaces, [22]. However, as with all indirect force control schemes, even low contact forces may lead to unacceptable positional errors, depending on parameter set and scenario. In [23] a disturbance observer is used to achieve convergence of the positional tracking error for null space impedance control in simulation.

In our contribution, we use direct force control, which gives us the ability to ensure low contact forces by all means and to keep exact positional tracking below a certain force threshold. The approach can be compared with a hybrid force/motion control scheme with adaptive selection of the spaces for force- and motion-control. For low contact forces, force control exploits the redundancy of the manipulator and motion control is carried-out in the task space. In the case of rising contact forces, force control is extended to both null space and task-space. The approach can be applied to the whole manipulator arm and not only to the end-effector.

\section{Environment Stiffness Estimation}

For current industrial applications in very structured and simple environments, it is sufficient to assume constant environment parameters for most interaction tasks. If the environment is unstructured or unknown however, this may lead to poor performance or even stability issues. Individual objects may have very different mechanical properties, which can not be detected with a pure vision-based approach. There exist several quite complex approaches in the field of online stiffness estimation based on force feedback, [24][28]. The approach presented in this paper provides a simple and efficient solution for online-stiffness estimation in the one-dimensional contact space. Experiments proof the applicability of this method even in demanding scenarios.

\section{TACTILE FEedBACK CONTROL FramewORK}

This section briefly recapitulates the control framework, which has been introduced in our previous work [1].

\section{A. General Framework}

We define the joint-space $\mathcal{C}$ as $\boldsymbol{q} \in \mathbb{R}^{n}$ and the task-space $\mathcal{W} \in \mathbb{R}^{m}$ as the end-effector position $\boldsymbol{w}:=[x, y, z]^{T}, m=3$, while arbitrary task-space definitions are possible as well. The null space $\mathcal{N}$ is the redundant space of the manipulator with $n>m$. Our control framework uses the tactile feedback controller inputs $\boldsymbol{u}_{j, q}, \boldsymbol{u}_{t, w}$ and $\boldsymbol{u}_{n, q}$ in joint-space, taskspace and null space based on the Automatic Supervisory Control scheme [29]:

$$
\begin{gathered}
\dot{\boldsymbol{q}}=\boldsymbol{J}_{w}^{\#}\left(\dot{\boldsymbol{w}}_{d, \mathrm{eff}}-\boldsymbol{u}_{t, w}\right)-\boldsymbol{u}_{j, q} \\
-\boldsymbol{N}_{w}\left(\boldsymbol{u}_{n, q}+\alpha\left(\frac{\partial H}{\partial \boldsymbol{q}}\right)^{T}\right) \\
\dot{\boldsymbol{w}}_{d, \mathrm{eff}}=\dot{\boldsymbol{w}}_{d}+\beta\left(\boldsymbol{w}_{d}-\boldsymbol{w}\right)
\end{gathered}
$$

with the weighted pseudoinverse $\boldsymbol{J}_{w}^{\#}$, the effective desired task-space velocity $\dot{\boldsymbol{w}}_{d \text {,eff }}$ (compensating numerical drift using the desired task-space position $\boldsymbol{w}_{d}$ and actual position $\boldsymbol{w}$ ), the null space projection matrix $\boldsymbol{N}_{w}$, the gains $\alpha, \beta$ and the gradient of a cost function $H$. The actual position $\boldsymbol{w}$ is calculated from the desired configuration $\boldsymbol{q}$ by solving forward kinematics. $H$ represents secondary objectives such as self-collision and joint-limit avoidance in null space [30]. Contacts with the environment are assumed to be frictionless using a linear-elastic material law.

In [1] we showed the transformation of the motion equation into the space along the contact force $F_{p}$ (in the following referred to as contact space) and derived the scalar equation

$$
\dot{F}_{p}=-c \dot{x}_{p, d, \text { eff }}+c k_{t}(t) u_{t}+c k_{j}(t) u_{j}+c k_{n}(t) u_{n}
$$

with the scalar controller inputs $u_{\{n, j, t\}}$ and the expected stiffness $c$ (cp. APPENDIX, (16) - (19)). Applying a feedback linearization scheme, tactile feedback controllers with 
arbitrary target system dynamics can be derived easily. The approach can also be used for acceleration-level inverse kinematics, commonly known as Resolved Acceleration Control [31].

\section{B. Controller Design}

For the system target dynamics of the controllers in this paper, we propose a linear second order behavior

$$
\ddot{\Xi}=-\frac{2 d}{T} \dot{\Xi}-\frac{1}{T^{2}} \Xi
$$

which describes a decrease of the contact force to zero with time constant $T$ and damping $d$. A first order dynamics leads to a non-zero steady-state error in the contact force and showed poorer performance compared to a second order target dynamics.

The proposed control framework allows several variants of tactile feedback controllers: An obvious approach is to choose controllers, which only use one system input $u_{\{n, j, t\}}$. A second order null space feedback controller $u_{n}$ has already been presented in our previous contribution [1]. The design for task-space $u_{t}$ and joint-space $u_{j}$ results in the following general control law:

$$
\begin{aligned}
u_{\{n, j, t\}}= & \frac{1}{k_{\{n, j, t\}}(t)} \dot{x}_{p, d, \mathrm{eff}}-\frac{2 d}{T c k_{\{n, j, t\}}(t)} F_{p} \\
& -\frac{1}{T^{2} c k_{\{n, j, t\}}(t)} \int_{0}^{t} F_{p} \mathrm{~d} \tau .
\end{aligned}
$$

All controllers contain the desired velocity of the contact point $\boldsymbol{p}$ in form of a feedforward term and depend on the scaling factors $k_{\{n, j, t\}}$ (see (16) to (19)), the expected stiffness $c$ and the parameters of the target dynamics $T$ and $d$. Control law extensions, e.g. limitation of the null space controller output, apply as presented in our previous publication [1]. In the following, we present the joint-space only controller with second order target system dynamics. The utilization of multiple system inputs is described in Sec. V. A task-space only controller overrides the desired trajectory in all cases to ensure minimal forces, which has not shown any benefit compared to the joint-space variant. Hence, it is not considered within this paper.

\section{JOINT-SPACE TACTILE FEEDBACK}

The control law for the joint-space only controller with second order dynamics is given by (5). The controller is not limited to the null space and reduces contact forces with the environment altering the desired trajectory of the manipulator. Still, it can be shown that the joint-space controller does implicitly make use of null space movements. To show this, we transform $(5)\left(u_{j}\right)$ into the vectorial representation, and insert the resulting $\boldsymbol{u}_{j, q}$ into (1) with $\boldsymbol{u}_{n, q}=\boldsymbol{u}_{t, w}=0$, $\alpha=0$. This yields

$$
\dot{\boldsymbol{q}}=\underbrace{\left(\boldsymbol{I}-\frac{\boldsymbol{J}_{p}^{T} \hat{\boldsymbol{F}}_{p} \hat{\boldsymbol{F}}_{p}^{T} \boldsymbol{J}_{p}}{k_{j}(t)}\right)}_{\boldsymbol{S}_{w}} \boldsymbol{J}_{w}^{\#} \underbrace{\left(\dot{\boldsymbol{w}}_{d}+\beta\left(\boldsymbol{w}_{d}-\boldsymbol{w}\right)\right)}_{\dot{\boldsymbol{w}}_{d, \mathrm{eff}}}
$$

$$
+\underbrace{\boldsymbol{J}_{p}^{T} \hat{\boldsymbol{F}}_{p}}_{\boldsymbol{S}_{f}} \underbrace{\left(\frac{2 d}{T c k_{j}(t)} F_{p}+\frac{1}{T^{2} c k_{j}(t)} \int_{0}^{t} F_{p} \mathrm{~d} \tau\right)}_{F_{c}}
$$

with the Jacobian $J_{p}$ at the contact point and the normalized contact force $\hat{\boldsymbol{F}}_{p}$. We observe that the force controller output $F_{c}$ is projected into the contact space with $\boldsymbol{S}_{f}$, while the effective task-space velocity $\dot{\boldsymbol{w}}_{d \text {,eff }}$ is projected into the remaining space, as $\boldsymbol{S}_{w}$ contains all directions minus the contact space. For a redundant robot, the solution is under-determined and the drift compensation with gain $\beta$ permanently corrects deviations from the desired task-space position, with exclusion of the contact-space. This means that the null space of the manipulator is used as long as this is sufficient to reduce the contact force. To achieve good utilization of null space movements, the drift compensation gain $\beta$ is set to higher values for joint-space controllers. Whereas with the null space variant the desired trajectory has a higher priority than the contact force control, the jointspace variant inverses the priorities with highest priority to zero contact forces.

\section{Multi-Space Tactile Feedback}

As considered in Sec. I, the task-space trajectory should have higher priority than the contact force control as long as the contact force stays below the threshold $F_{\text {act }}$, and lower priority as soon as it exceeds the threshold. Therefore, we propose the simultaneous utilization of multiple spaces, e.g. null space/joint-space or null space/task-space aiming at a flexible prioritization between force control and position tracking. Both variants work in general, however, we concentrate on the combination null space/task-space controller, since the respective spaces are orthogonal and therefore decoupled. The multi-space controller is designed hierarchically: as long as $F_{p} \leq F_{\text {act }}$ solely the null space controller is active. If $F_{p}>F_{\text {act }}$, the task-space controller is enabled and the manipulator deviates from the desired motion to reach $F_{p} \rightarrow F_{\text {act }}$.

\section{A. Controller Design}

While the design of the standalone null space/task-space controller has been presented in Sec. III, the combination of the two controllers requires some modifications to the task-space control law. We remove the feedforward term to avoid actuation in task-space for contact forces below $F_{\text {act }}$. Additionally, the rest position of the controller is shifted to $F_{\text {act }}$, which yields

$$
\begin{aligned}
u_{t}= & -\frac{2 d}{T c k_{t}(t)} \frac{1}{2}\left(1+\operatorname{sgn}\left(F_{p}-F_{\text {act }}\right)\right)\left(F_{p}-F_{\text {act }}\right) \\
& -\frac{1}{T^{2} c k_{t}(t)}\left(\int_{t_{a}}^{t_{b}} F_{p}-F_{\text {act }} \mathrm{d} \tau\right) .
\end{aligned}
$$

Application of the sgn function ensures that the controller is inactive in case the contact force is below $F_{\text {act }}$. In addition, the limits for the integral are changed with $t_{a}$ representing any point in time where $F_{p}>F_{\text {act }}$ becomes true and $t_{b}$ representing any point in time $\left(t_{b}>t_{a}\right)$ where the integral 
itself is reduced to zero by $F_{p}-F_{\text {act }}<0$. This ensures the integral part of the controller is only active if the force is above the activation threshold as well as the value of the integral never getting negative after the force dropped below the threshold. Furthermore, a smooth transition for the controller output between $F_{p}>F_{\text {act }}$ and $F_{p} \leq F_{\text {act }}$ is achieved.

\section{B. Stability}

The two controllers described by $(5)\left(u_{n}\right)$ and (7) are calculated simultaneously and applied to their respective inputs in the motion equation (1). Currently, we use the same target dynamics parameters for both controllers. This may be changed to account for the different behavior of the respective control spaces. The resulting system dynamics can be obtained by inserting the controller equations into (3) and differentiating with $\dot{F}_{\text {act }}=0$ to get:

$$
\ddot{F}_{p}= \begin{cases}-\frac{2 d}{T} \dot{F}_{p}-\frac{1}{T^{2}} F_{p}, & \text { if } A \\ -\frac{2 d}{T} \dot{F}_{p}-\frac{1}{T^{2}} F_{p}-\frac{1}{T^{2}}\left(F_{p}-F_{\text {act }}\right), & \text { if } B \\ -\frac{4 d}{T} \dot{F}_{p}-\frac{2}{T^{2}} F_{p}+\frac{1}{T^{2}} F_{\text {act }}, & \text { if } C\end{cases}
$$

with the boolean expressions

$$
\begin{aligned}
& A:=\left\{\left(F_{p} \leq F_{\text {act }}\right) \wedge\left(t<t_{a} \vee t \geq t_{b}\right)\right\} \\
& B:=\left\{\left(F_{p} \leq F_{\text {act }}\right) \wedge\left(t_{a} \leq t<t_{b}\right)\right\} \\
& C:=\left\{F_{p}>F_{\text {act }}\right\} .
\end{aligned}
$$

For $F_{p} \leq F_{\text {act }}$, the resulting dynamic is equal to our stable linear target dynamics, if the force threshold $F_{\text {act }}$ has not been exceeded $\left(t<t_{a}\right)$ or if the integral part is already zero again $t \geq t_{b}$. For $t_{a} \leq t<t_{b}$, an additional part caused by the integral term of the task-space controllers is active until the integral value becomes zero at $t=t_{b}$. As this happens in a finite time span, the system can still be considered stable. For $F_{p}>F_{\text {act }}$ both controllers are active and, in theory, this leads to different, but stable system dynamics under the constant disturbance $\frac{1}{T^{2}} F_{\text {act }}$. Still, the resulting system behavior is not asymptotically stable. In practice however, with an adequately high setting for $F_{\text {act }}$, the operation of the controllers is mutually excluded as the task-space controller has no influence below $F_{\text {act }}$ and the null space controller is already completely constrained by the joint limits if the contact forces rises above $F_{\text {act }}$. This means, the desired stable target dynamics with changed restposition $F_{\text {act }}$ is also reached for $F_{p}>F_{\text {act }}$.

\section{ONLINE ENVIRONMENT STIFFNESS ESTIMATION}

In the following, we present an online stiffness estimation, which is used to adapt the parameters of the control laws. However, the algorithm works independently and can be used for arbitrary purposes.

\section{A. Effects of Unknown Environment Stiffness}

The tactile feedback control laws contain the expected environment stiffness $c$, which may differ from the actual environment stiffness $c_{e}$. To analyze the resulting effects, we replace $c=c_{e}$ in the contact force equation (3), insert the controller equation (5) (for one space), and differentiate to obtain

$$
\ddot{F}_{p}=-\frac{2 d c_{e}}{T c} \dot{F}_{p}-\frac{c_{e}}{T^{2} c} F_{p}
$$

For $c=c_{e}$, this matches the desired target dynamics (4) with $\Xi(t)=F_{p}(t)$. Otherwise, the changed system dynamics can be written as a second-order system with the effective timeconstant

$$
T_{\mathrm{eff}}=T \sqrt{\frac{c}{c_{e}}} \quad \text { and damping } \quad d_{\mathrm{eff}}=d \sqrt{\frac{c_{e}}{c}} .
$$

The overall influence of the relation $c_{e} / c$ can be summarized w.r.t. the target dynamics:

$$
\begin{aligned}
& c_{e}>c \Rightarrow \text { faster dynamics, higher damping } \\
& c_{e}<c \Rightarrow \text { slower dynamics, lower damping. }
\end{aligned}
$$

In general, this may be considered beneficial, as the dynamics are faster for stiffer objects and slower for softer objects. However, the lower time-constant with faster dynamics might lead to instability of the system. Therefore, $c \geq c_{e}$ has to hold for the chosen assumed stiffness. Consequently, the performance with very soft objects is bad, as a high difference to the actual environment stiffness slows down the system dynamics significantly.

\section{B. Online Estimation Algorithm}

To overcome this issue, we propose a simple algorithm for online environment stiffness estimation. As we assume a linear-elastic material, $c_{e}$ can be estimated using

$$
c_{e} \approx c=\frac{\Delta F_{p}}{\Delta x_{p}}
$$

with a change in the contact force $\Delta F_{p}$ and the corresponding traveled distance $\Delta x_{p}$ along the contact space. We further assume $\Delta x_{p} \approx \Delta x_{p, d}$, i.e. the desired movement of the manipulator is used. This seems reasonable for a positioncontrolled robot in a static environment and avoids noise caused from the sensor signals. The traveled distance of the contact point is obtained by integration of the desired velocity in contact space

$$
v_{p, d}=-\hat{\boldsymbol{F}}_{p}^{T} \boldsymbol{J}_{p} \dot{\boldsymbol{q}}_{d}
$$

To account for a limited accuracy of the tactile sensor, the estimation is performed only if the contact force rises above the fixed threshold $\Delta F_{\text {est,min }}$. In addition, the algorithm is deactivated once the manipulator begins to move away from the environment. This is because the return path of the contact point may not be the same as the original path, which leads to residual distances in the integrator causing wrong estimations. To get a continuous update of the estimated stiffness, we trigger the estimation every time the contact force increases by $\Delta F_{\text {est,min }}$. Thereby, changes in the environment stiffness during contact with the environment are detected. Alg. 1 summarizes the proposed approach. 


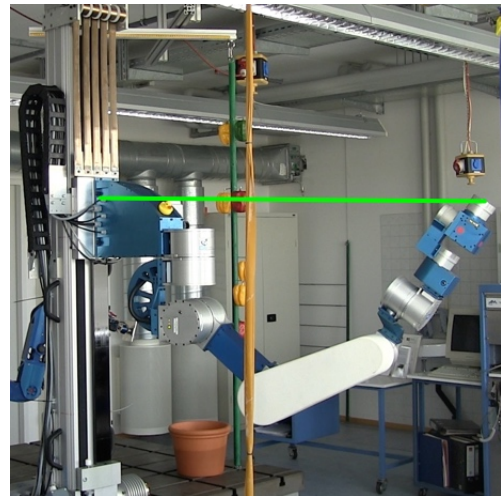

(a) Scenario 1 with latex-band

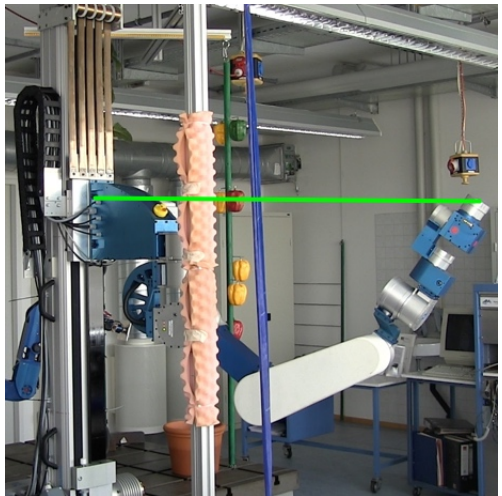

(b) Scenario 2 with low stiffness latex-band and aluminum profile

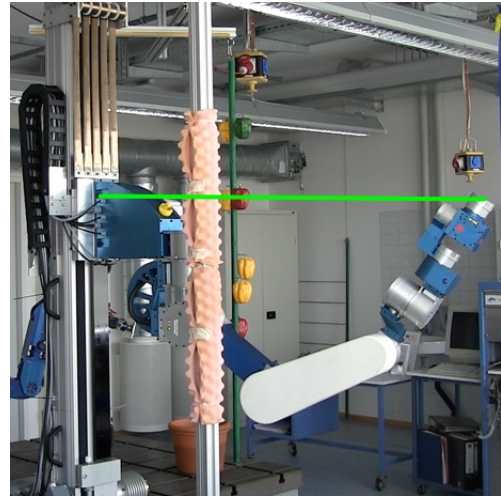

(c) Scenario 3 with aluminum profile

Fig. 1: In the conducted experiments, our 9-DOF manipulator hits different obstacles while following a straight line in $\mathcal{W}$-space with the end-effector (green line, from right to left)

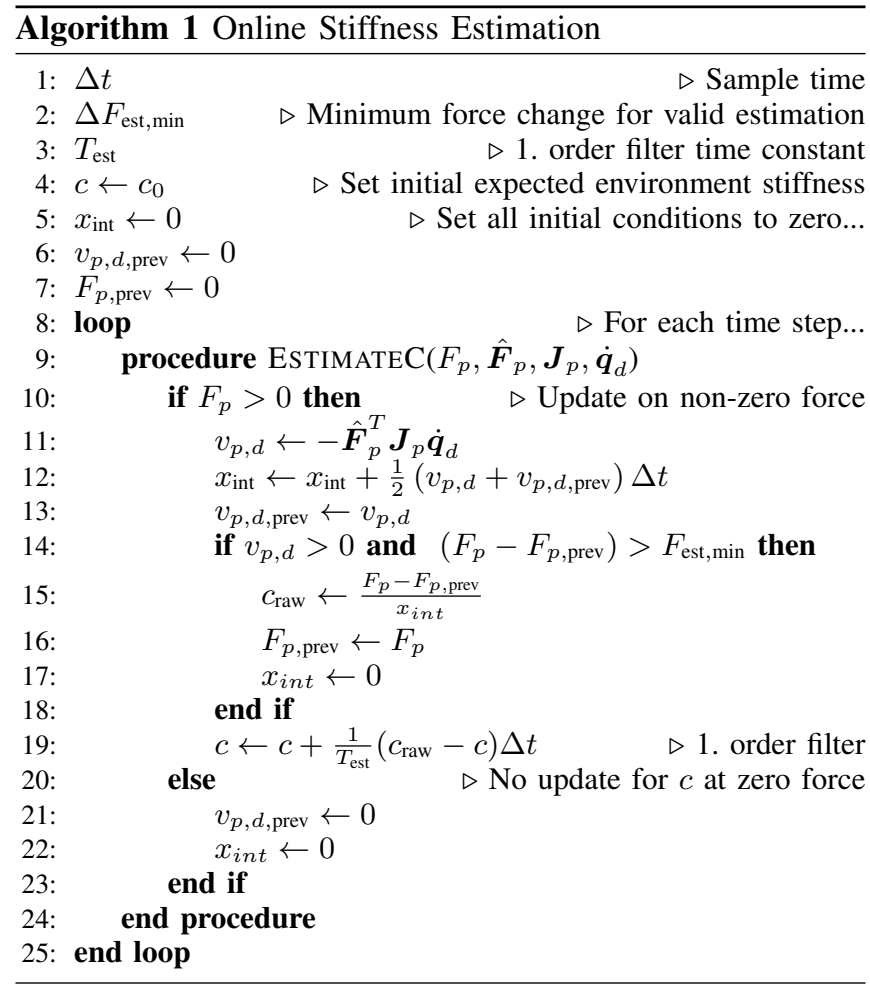

\section{RESULTS}

We conducted several experiments in three different collision scenarios, where motion planning without tactile feedback leads to high contact forces with the environment and even pure null space controllers can not reduce the force to zero:

1) The manipulator hits a pretensioned, elastic vertical latex-band with stiffness $c_{e, \mathrm{lt} 1}$ at the contact point while performing a horizontal movement, see Fig. 1a.

2) A pretensioned, elastic vertical latex-band with stiff-

\begin{tabular}{ll}
\hline $\begin{array}{l}\text { Control Law Update Rate } \\
\text { Null Space Gain } \alpha\end{array}$ & $1 \mathrm{~ms}$ \\
$\begin{array}{l}\text { Drift Compensation Gain } \beta \\
\quad \text { for joint-space controllers, see Sec. IV }\end{array}$ & 500 \\
otherwise & 5 \\
Controller Time Constant $T$ & $0.1 \mathrm{~s}$ \\
Controller Damping $d$ & 0.5 \\
Assumed Environment Stiffness $c_{0}$ & $7000 \mathrm{~N} / \mathrm{m}$ \\
Controller Activation Threshold $F_{t h}$ & $1.2 \mathrm{~N}$ \\
Multi-Space Activation Threshold $F_{\text {act }}$ & $5 \mathrm{~N}-F_{t h}=3.8 \mathrm{~N}$ \\
Null Space Velocity Limitation & \\
$\quad$ for prismatic joints $\left|q_{\text {prism,max }}\right|$ & $5 \mathrm{~m} / \mathrm{s}$ \\
$\quad$ for revolute joints $\left|q_{\mathrm{rot}, \max }\right|$ & $5 \mathrm{rad} / \mathrm{s}$ \\
Contact Transition Max. Time Constant $T_{t, \max }$ & $1 \mathrm{~s}$ \\
Contact Transition Distance $d_{t}$ & $0.05 \mathrm{~m}$ \\
Online Estimation Force Threshold $F_{\text {est,min }}$ & $0.2 \mathrm{~N}$ \\
Online Estimation Filter Time Constant $T_{\mathrm{est}}$ & $0.03 \mathrm{~s}$ \\
Stiffness Latex-band 1 (golden) $c_{e, \mathrm{lt} 1}$ & $\approx 60 \mathrm{~N} / \mathrm{m}$ \\
Stiffness Latex-band 2 (blue) $c_{e, \mathrm{lt} 2}$ & $\approx 30 \mathrm{~N} / \mathrm{m}$ \\
Stiffness Aluminum Profile $c_{e, \text { alu }}$ & $\approx 17000 \mathrm{~N} / \mathrm{m}$ \\
\hline
\end{tabular}

TABLE I: Parameters of the control laws and the environment

ness $c_{e, 1 \mathrm{t} 2}$ and a vertical solid aluminum profile $^{1}$ $\left(c_{e, \text { alu }}\right)$, which is fixed at its top and bottom, are hit, see Fig. $1 b$.

3) The latex-band is removed, solely the vertical aluminum profile obstructs the manipulator, see Fig. 1c.

In all scenarios, the end-effector of the manipulator had to follow a straight line in $\mathcal{W}$-space. The parameters for the control laws and the scenarios are summarized in Tab. I. The initial stiffness $c_{0}$ is an estimation for the stiffness of the manipulator and therefore an upper limit for the overall stiffness of the contact. We chose this upper limit to ensure stability according to Sec. VI-A.

\section{A. Hardware Platform}

The tactile planning approach presented above has been implemented on a 9-DOF manipulator equipped with one tactile sensor. The manipulator has been developed for the

\footnotetext{
${ }^{1}$ The aluminum bar is wrapped with deformable foam material to avoid damages to the tactile cover from tangential movements.
} 


\begin{tabular}{ll}
\hline Range & $F_{\text {sen }}=1.5-20 \mathrm{~N}$ \\
Accuracy & $<0.1 \mathrm{~N}$ \\
Update Rate Force & $<4 \mathrm{~ms}$ \\
Filtering Force Signal & $\omega_{c}=15 \mathrm{~Hz}$, Butterworth $3^{\text {rd }}$ order \\
Force Sensors & $8 \times$ HONEYWELL FSS020WNGXS \\
Analog Digital Converter & $2 \times$ ANALOG DEVICES AD7738, 24 Bit \\
Controller & ATMEL AT90CAN128 \\
CAN Bandwidth & $1 \mathrm{Mbps}$ \\
\hline
\end{tabular}

TABLE II: Specifications of the Tactile Sensor, cp. [1]

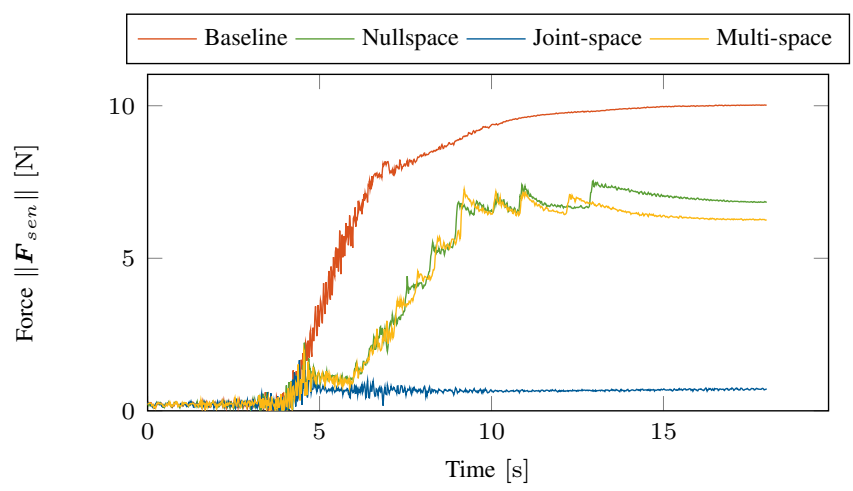

Fig. 2: Scenario 1 - Measured contact force on the tactile sensor during collision of the manipulator without force controller (red), with the standalone null space (green) and joint-space (blue) controllers and the multi-space controller (orange).

multipurpose use in agricultural tasks (e.g. selective harvesting of sweet-pepper, apples; precision spraying of grapes) within the EU-project CROPS $^{2}$ [32]. It has one prismatic and 8 revolute joints, $n=9$. In [1] we introduced the tactile sensing module, which is mounted at link 4 for measuring external torques and forces. Its main specifications are summarized in Tab. II. Contact force components tangential to the surface of the tactile cover are filtered out, as friction is not considered in our contact model. The measured torques are used to compute the contact point $\boldsymbol{p}$, which is fixed on the connection line between link 4 and 5 and only variable in the longitudal direction of the tactile sensing module.

\section{B. Scenario 1 - Soft Latex Band}

Evaluation of the controllers in Scenario 1 (Fig. 1a) shows high contact forces without force controller (Baseline) and significantly reduced contact forces with tactile feedback (Fig. 2). The null space controller is not able to reduce the force to zero, instead it remains at $6.8 \mathrm{~N}$. The peaks in the force signal are caused by stick-slipping of the latexband at the tactile cover, which can be seen in our attached videos. The joint-space controller reduces the contact force to $0.7 \mathrm{~N}$ which is even below the activation threshold $F_{t h}=$ $1.2 \mathrm{~N}$. Because of the Continuous Feedforward Compensation extension described in [1], the desired trajectory is even abandoned for $F_{\text {sen }}<F_{t h}$ and further movement in direction

\footnotetext{
${ }^{2}$ CROPS: Clever Robots for Crops, European Project within the 7th Framework Program, 10/2010-09/2014, GA 246252.
}

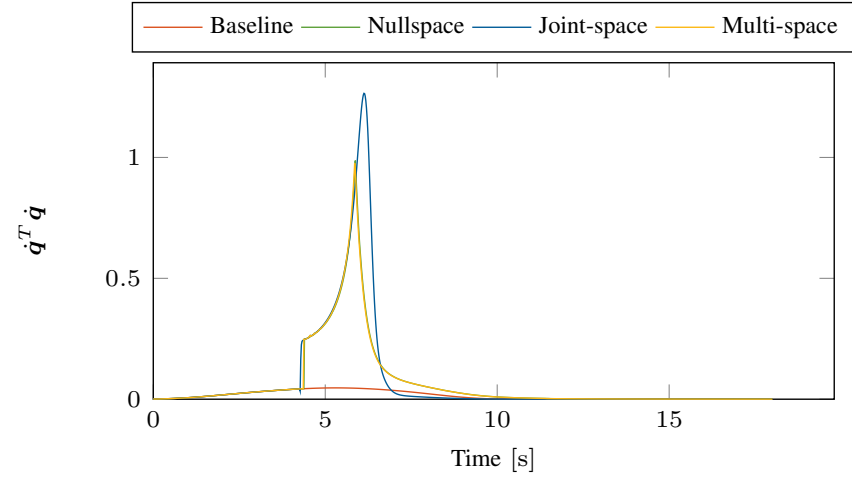

Fig. 3: Scenario 1 - Squared joint velocities during collision without force controller (red), using the standalone null space (green) and joint-space controllers (blue) and the multi-space controller (orange).

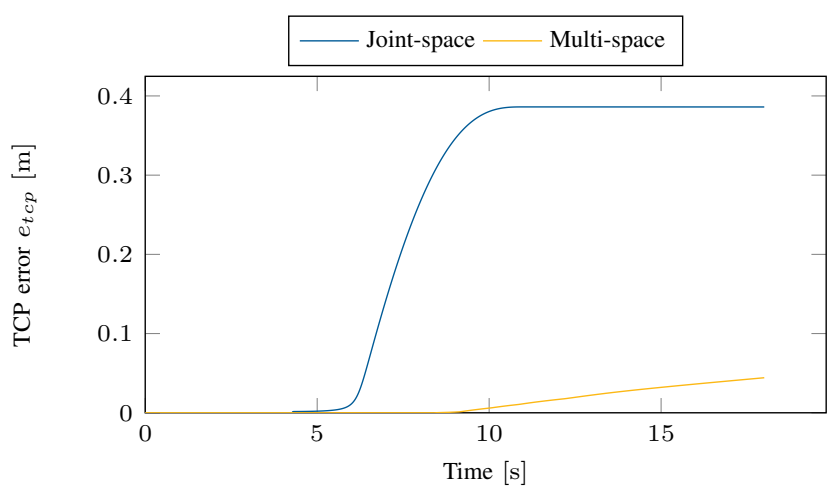

Fig. 4: Scenario 1 - Deviation of the end-effector (TCP) from the desired trajectory using the joint-space (blue) and multispace controller (orange).

of the obstacle is avoided. The joint-space controller results in the highest joint velocities, see Fig. 3. The multi-space controller does not reduce the contact force to the target of $5 \mathrm{~N}$ within the considered time interval, but the deviation of the end-effector slowly increases over time (Fig. 4). Note that the end-effector deviation is zero with pure nullspace movement until the force threshold $F_{\text {act }}$ is exceeded. The dynamics of the task-space part of the multi-space controller is too slow in this case, which is caused by the high difference between the assumed and the actual environment stiffness, refer to Sec. VI-A. Compared to the aggressive reaction of the joint-space controller, the deviation from the desired trajectory is performed gradually.

\section{Scenario 2 - Latex Band and Aluminum Profile}

Because of its flexibility, the multi-space controller can also be used in the more complex Scenario 2 (Fig. 1b) with high difference in the stiffness of the individual objects. Without online stiffness estimation, the contact force slowly rises after first contact with the latex-band at $t=2 \mathrm{~s}$, then the aluminum profile is reached at $t=8 \mathrm{~s}$ and the contact force increases to a maximum of $16.8 \mathrm{~N}$ before the controller slowly reaches the desired contact force of $5 \mathrm{~N}$ 


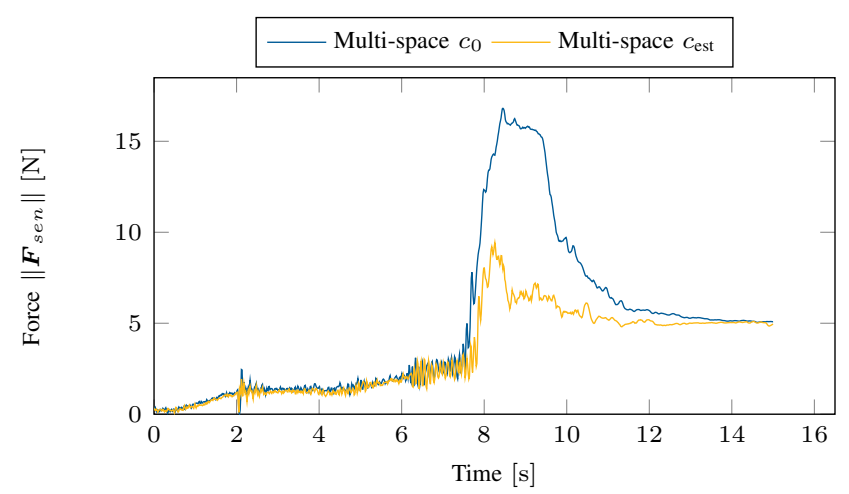

Fig. 5: Scenario 2 - Measured contact force using the multispace controller without (blue) and with (orange) online stiffness estimation

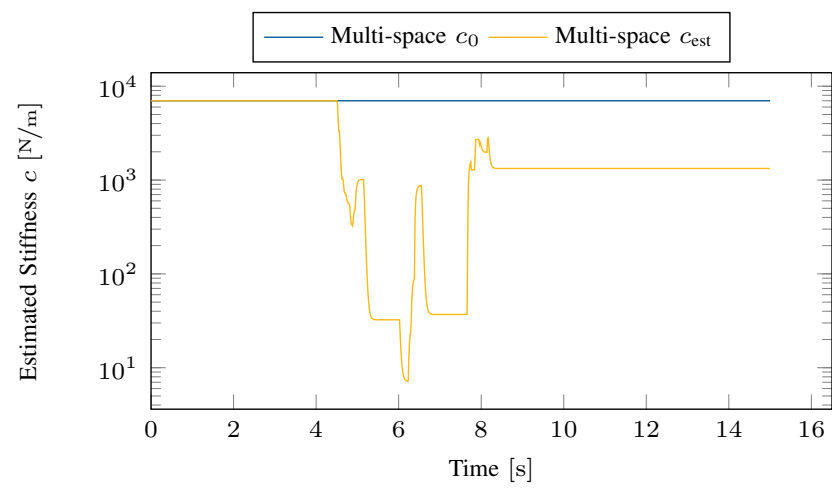

Fig. 6: Scenario 2 - Assumed (blue) and estimated (orange) environment stiffness using the multi-space controller.

(Fig. 5). Compared to the experiments with the sole latexband, the multi-space controller reaches its desired target contact force, as the difference between assumed $c_{0}$ and actual $c_{e}$ environment stiffness is lower and the effective dynamics more similar to the original target dynamics, see Sec. VI-A. The TCP error in this experiment is about $0.08 \mathrm{~m}$.

With activated online stiffness estimation, a much faster behavior is reached as the environment stiffness is below the assumed stiffness. This results in a lower maximum contact force of $9.4 \mathrm{~N}$ and faster decrease to $F_{p}=F_{\text {act }}$. Fig. 6 depicts the estimated stiffness for this scenario. After first contact, the estimation reduces the stiffness to $32 \mathrm{~N} / \mathrm{m}$, which is fairly near to the actual stiffness $c_{e, \mathrm{tt} 2} \approx 30 \mathrm{~N} / \mathrm{m}$ of the band. At $t \approx 6 \mathrm{~s}$ unexpected changes of the estimated stiffness occur, which is due to oscillations of the latex band. These trigger the stiffness estimation due to amplitudes in the measured force greater than $F_{\text {est } \min }=0.2 \mathrm{~N}$. At $t=8 \mathrm{~s}$, the aluminum profile with its foam coating is reached and the estimation increases rapidly. Shortly after the peak, the estimation is shut off as the manipulator is no longer moving in the direction of the obstacle.

\section{Scenario 3 - Aluminum Profile}

To demonstrate the high performance and flexibility of our solution, the multi-space controller is used in the third

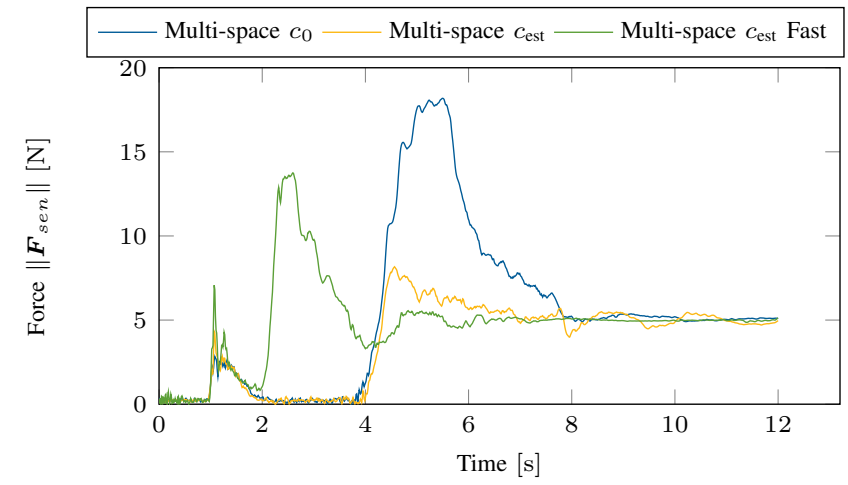

Fig. 7: Scenario 3 - Measured contact force using the multispace controller without (blue), with (orange) online stiffness estimation, and higher $\mathcal{W}$-space velocity (green).

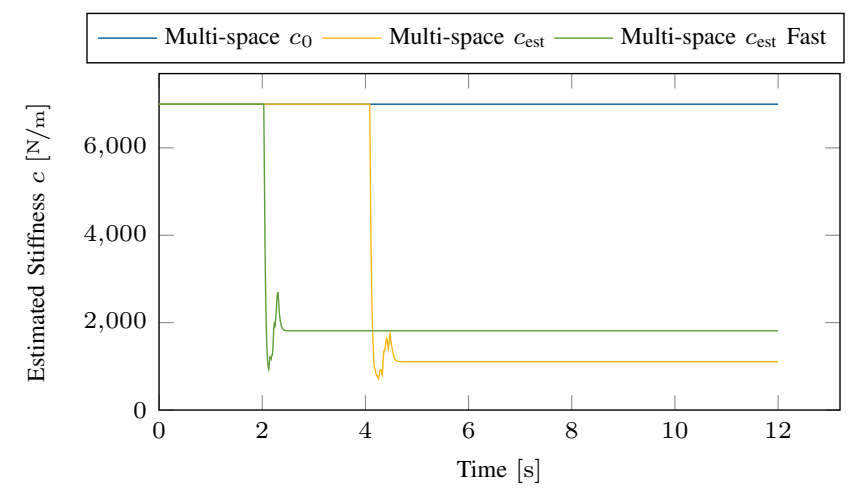

Fig. 8: Scenario 3 - Assumed (blue), and estimated environment stiffness for slow (orange) and fast (green) movement.

scenario to move against the stiff aluminum profile without hitting a soft object before. Fig. 7 depicts the contact forces for three different variants. The first only uses $c_{0}$, the second is with stiffness estimation and the third is with stiffness estimation and a desired $\mathcal{W}$-space velocity, which is about 2.4 times higher. Again, the online stiffness estimation results in a significant performance increase with a decrease of the maximum contact force by $55 \%$. Even with the higher $\mathcal{W}$ space velocity, the maximum contact force stays below the value for the controller without stiffness estimation. The absolute estimated stiffnesses for slow and fast movement against the obstacle are different (Fig. 8). As the estimation averages over the stiffness of the foam coating and the aluminum profile itself, and higher speeds lead to farther movements against the obstacle, the estimation is higher for faster speeds as the average contains a higher share of the stiffness of the profile than the foam coating.

\section{CONCLUSION}

In our contribution we presented significant extensions to our tactile feedback control approach published in [1]. This includes the design of a joint-space based tactile feedback controller, which is not limited to the null space and may directly alter the motion of the manipulator to achieve minimal contact forces during collisions with the environment. 
Additionally, the concept of multi-space controllers was introduced to provide flexible prioritization between low contact forces and good positional tracking of the endeffector. Using this technique, a given task in $\mathcal{W}$-space is only abandoned if the contact forces exceed a certain limit. This prevents from damaging the manipulator or the environment, while the task is still carried out as good a possible. Below the force limit, the redundant space of the robot is used to reduce contact forces with the environment. We were able to show the effectiveness and practicability of our smooth controller transition at the force threshold in real-world experiments.

Furthermore, we presented an online stiffness estimation algorithm to reach higher performances with the described tactile feedback controllers. The estimated stiffness might also be used for other purposes as well. Finally, we conducted several experiments on our multipurpose 9-DOF manipulator with tactile sensing module and could show a high performance during collision with very soft (latex-band) as well as very stiff (aluminum profile) objects.

\section{APPENDIX}

The following equations are used for the transformation into the contact-space and back into the $\mathcal{C}$-space and have been derived in [1]:

$$
\begin{aligned}
\boldsymbol{F}_{p} & =\hat{\boldsymbol{F}}_{p}\left\|\boldsymbol{F}_{p}\right\|=\hat{\boldsymbol{F}}_{p} F_{p} & \boldsymbol{u}_{n, q} & =\boldsymbol{J}_{p}^{T} \hat{\boldsymbol{F}}_{p} u_{n} \\
\boldsymbol{u}_{j, q} & =\boldsymbol{J}_{p}^{T} \hat{\boldsymbol{F}}_{p} u_{j} & \boldsymbol{u}_{t, w} & =\boldsymbol{J}_{w} \boldsymbol{J}_{p}^{T} \hat{\boldsymbol{F}}_{p} u_{t} \\
\dot{x}_{p, d, \mathrm{eff}} & :=\hat{\boldsymbol{F}}_{p}^{T} \boldsymbol{J}_{p} \boldsymbol{J}_{w}^{\#} \dot{\boldsymbol{w}}_{d, \mathrm{eff}} & k_{j}(t) & :=\hat{\boldsymbol{F}}_{p}^{T} \boldsymbol{J}_{p} \boldsymbol{J}_{p}^{T} \hat{\boldsymbol{F}}_{p} \\
k_{n}(t) & :=\hat{\boldsymbol{F}}_{p}^{T} \boldsymbol{J}_{p} \boldsymbol{N}_{\boldsymbol{w}} \boldsymbol{J}_{p}^{T} \hat{\boldsymbol{F}}_{p} & k_{t}(t) & :=\hat{\boldsymbol{F}}_{p}^{T} \boldsymbol{J}_{p} \boldsymbol{J}_{w}^{\#} \boldsymbol{J}_{w} \boldsymbol{J}_{p}^{T} \hat{\boldsymbol{F}}_{p}
\end{aligned}
$$

\section{REFERENCES}

[1] C. Schuetz, J. Pfaff, F. Sygulla, D. Rixen, and H. Ulbrich, "Motion Planning for Redundant Manipulators in Uncertain Environments based on Tactile Feedback," in Proc. IEEE/RSJ International Conference on Intelligent Robots and Systems, 2015.

[2] T. Lozano-Perez, "Spatial Planning: A Configuration Space Approach," IEEE Transactions on Computers, vol. C-32, no. 2, pp. 108$120,1983$.

[3] O. Khatib, "Real-time obstacle avoidance for manipulators and mobile robots," The international journal of robotics research, vol. 5, no. 1, pp. 90-98, 1986.

[4] J. J. Kuffner and S. M. LaValle, "RRT-connect: An efficient approach to single-query path planning," in Proc. IEEE International Conference on Robotics and Automation, vol. 2, 2000, pp. 995-1001 vol.2.

[5] N. Ratliff, M. Zucker, J. A. Bagnell, and S. Srinivasa, "CHOMP: Gradient optimization techniques for efficient motion planning," in Proc. IEEE International Conference on Robotics and Automation, 2009, pp. 489-494.

[6] J. Schulman, J. Ho, A. Lee, I. Awwal, H. Bradlow, and P. Abbeel, "Finding Locally Optimal, Collision-Free Trajectories with Sequential Convex Optimization." in Proc. of Robotics: Science and Systems, 2013.

[7] M. Dogar and S. Srinivasa, "A framework for push-grasping in clutter," Robotics: Science and Systems VII, 2011.

[8] M. Stilman, J.-U. Schamburek, J. Kuffner, and T. Asfour, "Manipulation Planning Among Movable Obstacles," in Proc. IEEE International Conference on Robotics and Automation, 2007, pp. 3327-3332.

[9] B. Frank, C. Stachniss, N. Abdo, and W. Burgard, "Efficient motion planning for manipulation robots in environments with deformable objects," in Proc. IEEE/RSJ International Conference on Intelligent Robots and Systems, 2011, pp. 2180-2185.
[10] S. Patil, J. van den Berg, and R. Alterovitz, "Motion planning under uncertainty in highly deformable environments," Robotics: Science and Systems VII, 2011.

[11] I. D. Walker, "The use of kinematic redundancy in reducing impact and contact effects in manipulation," in Proc. IEEE International Conference on Robotics and Automation, 1990, pp. 434-439 vol.1.

[12] W. J. Chung, I. H. Kim, and J. Joh, "Null-space dynamics-based control of redundant manipulators in reducing impact," Control Engineering Practice, vol. 5, no. 9, pp. 1273-1282, 1997.

[13] M. W. Gertz, J.-O. Kim, and P. K. Khosla, "Exploiting redundancy to reduce impact force," in Proc. IEEE/RSJ International Conference on Intelligent Robots and Systems, 1991, pp. 179-184 vol.1.

[14] A. Jain, M. D. Killpack, A. Edsinger, and C. C. Kemp, "Reaching in clutter with whole-arm tactile sensing," The International Journal of Robotics Research, vol. 32, no. 4, pp. 458-482, 2013.

[15] M. D. Killpack and C. C. Kemp, "Fast Reaching in Clutter While Regulating Forces Using Model Predictive Control," in Proc. IEEERAS International Conference on Humanoid Robots, 2013.

[16] T. Bhattacharjee, P. M. Grice, A. Kapusta, M. D. Killpack, D. Park, and C. C. Kemp, "A Robotic System for Reaching in Dense Clutter that Integrates Model Predictive Control, Learning, Haptic Mapping, and Planning," in Proc. IEEE/RSJ International Conference on Intelligent Robots and Systems, 2014.

[17] L. Villani and J. De Schutter, "Force control," in Springer Handbook of Robotics. Springer, 2008, ch. 7, pp. 161-185.

[18] E. Magrini, F. Flacco, and A. D. Luca, "Control of Generalized Contact Motion and Force in Physical Human-Robot Interaction," Proc. IEEE International Conference on Robotics and Automation, pp. 2298-2304, 2015.

[19] J. Park and O. Khatib, "Robot multiple contact control," Robotica, vol. 26, no. 05, pp. 667-677, 2008.

[20] B. Nemec and L. Zlajpah, "Force control of redundant robots in unstructured environment," IEEE Transactions on Industrial Electronics, vol. 49, no. 1, pp. 233-240, 2002.

[21] C. Ott, A. Dietrich, and A. Albu-Schäffer, "Prioritized multi-task compliance control of redundant manipulators," Automatica, vol. 53, pp. 416-423, 2015.

[22] R. Platt, M. Abdallah, and C. Wampler, "Multiple-priority impedance control," Proc. IEEE International Conference on Robotics and Automation, 2011

[23] H. Sadeghian, M. Keshmiri, L. Villani, and B. Siciliano, "Nullspace impedance control with disturbance observer," Proc. IEEE/RSJ International Conference on Intelligent Robots and Systems, pp. 27952800, 2012.

[24] D. Erickson, M. Weber, and I. Sharf, "Contact Stiffness and Damping Estimation for Robotic Systems," The International Journal of Robotics Research, vol. 22, no. 1, pp. 41-57, 2003.

[25] N. Diolaiti, C. Melchiorri, and S. Stramigioli, "Contact impedance estimation for robotic systems," IEEE Transactions on Robotics, vol. 21, no. 5, pp. 925-935, 2005.

[26] R. Cortesao, J. Park, and O. Khatib, "Real-time adaptive control for haptic manipulation with Active Observers," Proc. IEEE/RSJ International Conference on Intelligent Robots and Systems, vol. 3, no. 5, pp. 987-999, 2003.

[27] F. Coutinho and R. Cortesão, "Environment stiffness estimation with multiple observers," Proc. Industrial Electronics Conference, pp. 1537-1542, 2009.

[28] F. Coutinho and R. Cortesao, "Comparison of position and force-based techniques for environment stiffness estimation in robotic tasks," Proc. IEEE/RSJ International Conference on Intelligent Robots and Systems, vol. 1, pp. 4933-4938, 2012.

[29] A. Liégeois, "Automatic Supervisory Control of the Configuration and Behavior of Multibody Mechanisms," IEEE Transactions on Systems, Man, and Cybernetics, vol. 7, no. 12, pp. 868-871, dec 1977.

[30] C. Schuetz, T. Buschmann, J. Baur, J. Pfaff, and H. Ulbrich, "Predictive Online Inverse Kinematics for Redundant Manipulators," in Proc. IEEE International Conference on Robotics and Automation, 2014, pp. 5056-5061.

[31] J. Y. S. Luh, M. W. Walker, and R. P. C. Paul, "Resolved-acceleration control of mechanical manipulators," IEEE Transactions on Automatic Control, vol. 25, no. 3, pp. 468-474, jun 1980.

[32] C. Schuetz, J. Pfaff, J. Baur, T. Buschmann, and H. Ulbrich, "A Modular Robot System for Agricultural Applications," in Proc. International Conference of Agricultural Engineering, 2014. 